\title{
Prevalence of High-Grade Cervical Dysplasia in an Inner City Adolescent Population
}

\author{
Kevin S. Smith • Vanessa J. McDonald • Babak Shokrani
}

Received: 10 October 2013 /Revised: 17 January 2014 / Accepted: 5 February 2014 /Published online: 24 May 2014

(C) Cobb/NMA Health Institute 2014

\begin{abstract}
Objective The purpose of this study was to determine the prevalence of high-grade squamous intraepithelial lesions (HSIL) in an adolescent population diagnosed at an urban university hospital.

Methods This study was a retrospective review of Pap smear diagnoses from January 1997 through August 2010. Patients $\leq 20$ years were compared with records of patients ages 21-30 and $>30$ years and also with US averages. Analyses were conducted using the Pearson chi-square test.

Results A total of 19,481 Pap smear diagnoses of AfricanAmerican patients were reviewed: 2,229 for patients $\leq 20$ years old, 5,294 for patients 21-30 years old, and 11,958 for patients $>30$ years old. The prevalence of HSIL in study patients $\leq 20$ year age group was higher $(1.62 \%)$ than that of the $21-30$ year age group $(1.38 \%)$ and the $>30$ year age group $(1.10 \%)$. The difference between the $\leq 20$ year age group and the $>30$ year age group was statistically significant $(\mathrm{p}=0.0478)$ whereas the difference betweeen the $\leq 20$ year age group and the 21-30 year age group was not $(p=0.458)$. Follow-up management of seventeen patients $\leq 20$ years old with HSIL indicated that eleven $(64.7 \%)$ patients had a persistent diagnosis of HSIL, four $(23.5 \%)$ patients had a diagnosis of low-grade squamous intraepithelial lesion (LSIL), and two (11.8\%) patients had no evidence of intraepithelial lesion or malignancy on subsequent colposcopy or conization.

Conclusion Our study data supports published reports of a high prevalence of HSIL in adolescents. However, the prevalence of malignant transformation in this cohort is still
\end{abstract}

\footnotetext{
K. S. Smith $(\bowtie) \cdot$ V. J. McDonald

Department of Obstetrics and Gynecology, Howard University Hospital, 2041 Georgia Avenue, Northwest Suite 3C03, Washington, DC 20060, USA

e-mail: K_Smith@howard.edu

B. Shokrani

Department of Pathology, Howard University Hospital, Washington, DC 20060, USA
}

relatively low. As such, we agree with current guidelines posited by the American College of Obstetrics and Gynecology (ACOG), American Cancer Society (ACS), and US Preventive Service Task Force (USPSTF). Room for additional research in the African-American cohort is encouraged given their underrepresentation in research.

Keywords Human papilloma virus · Adolescents · Cervical dysplasia $\cdot$ Pap smear $\cdot$ High grade squamous intraepithelial lesion $\cdot$ Low grade squamous intraepithelial lesion

\section{Introduction}

In 2009, the American College of Obstetrics and Gynecology (ACOG), American Cancer Society (ACS), and US Preventive Service Task Force (USPSTF) adopted the initiation of cervical cancer screening to 21 years of age, prompted by the supposition of a handful of research studies that the prevalence of invasive cancer was low in the adolescent population [1]. Only $0.1 \%$ of cases of cervical cancer occur before age 21 [2], which translates to approximately 1-2 cases per year per 1,000,000 females age 15-19 [2,3]. Although cancer is rare in adolescents, dysplasia is not. In a report of 10,090 Pap test results in females aged 12 18 years, 422 specimens $(5.7 \%)$ were reported as LSIL and 55 specimens $(0.7 \%)$ were HSIL [4].

In addition to the guidelines on the initiation of cervical cancer screening, ACOG, ACS, and USPSTF also have outlined recommended follow-up for various cytologic abnormalities. In adolescents with atypical squamous cells of undetermined significance (ASC-US), a follow-up with annual cytologic testing is recommended. At the 12-month follow-up, only adolescents with HSIL or greater on the repeat cytology should be referred to colposcopy. At the 24-month follow-up, those with an ASCUS or greater result should be referred to colposcopy. HPV DNA testing and colposcopy are unacceptable for adolescents 
with ASC-US. If HPV testing is inadvertently performed, the results should not influence management [1].

In adolescents with low-grade squamous intraepithelial lesion (LSIL), a follow-up with annual cytologic testing is recommended. At the 12-month follow-up, only adolescents with HSIL or greater on the repeat cytology should be referred to colposcopy. At the 24-month follow-up, those with an ASC-US or greater result should be referred to colposcopy. HPV DNA testing is unacceptable for adolescents with LSIL. If HPV DNA testing is inadvertently performed, the results should not influence management [6].

In adolescents with HSIL, colposcopy is recommended. When CIN 2 and CIN 3 are not identified histologically, observation for up to 24 months using both colposcopy and cytology at 6-month intervals is preferred, provided the colposcopic examination is satisfactory and endocervical sampling is negative. In exceptional circumstances, a diagnostic excisional procedure is acceptable. If, during follow-up, a high-grade colposcopic lesion is identified or HSIL cytology persists for 1 year, biopsy is recommended. If CIN 2 and CIN 3 are identified histologically, management should follow the 2006 Consensus Guidelines for the Management of Women with Cervical Intraepithelial Neoplasia. If HSIL persists for 24 months without identification of CIN 2 and CIN 3, a diagnostic excisional procedure is recommended. After two consecutive "negative for intraepithelial lesion or malignancy" results, adolescents and young women without a high-grade colposcopic abnormality can return to routine cytological screening. A diagnostic excisional procedure is recommended for adolescents and young women with HSIL when colposcopy is unsatisfactory or CIN of any grade is identified on endocervical assessment [1].

Although current recommendations support less invasive management, this may prove problematic in populations less likely to have access to care or have compliance issues such as adolescents and members of certain ethnic groups. Review of the literature reveals a paucity of data concerning this age group, and, to a lesser extent, those of African-American descent. We investigated a subset of unique adolescents not specified by the ACOG guidelines with the goal of determining whether current revised guidelines were appropriate for said population.

\section{Materials and Methods}

We conducted a retrospective review of cytologic Pap smear results (via Thin Prep, Hologic, Inc., Marlborough, MA) at Howard University Hospital. All Pap smear records had been conducted between January 1997 and August 2010. Diagnoses of three age groups of primarily AfricanAmerican patients were reviewed for this time period: $\leq 20$ years of age, $21-30$ years of age, and $>30$ years of age. These particular age ranges were used due to the fact that each group has different risks for the development of premalignant and malignant cervical lesions, and therefore the recommendations for screening frequency and HPV cotesting are different in each one.

In March 2012, updated screening guidelines were released by the US Preventive Services Task Force jointly with the American Cancer Society, the American Society for Colposcopy and Cervical Pathology, and the American Society for Clinical Pathology. These guidelines recommend that women have their first Pap test at age 21 because adolescents have a very low risk of cervical cancer and a high likelihood that cervical cell abnormalities will spontaneously regress. The guidelines further advise that routine Pap smear and HPV cotesting be limited to women age 30 and older, because in older women, HPV infections are more likely to represent persistent infections that have the potential to progress to cervical cancer if not detected or treated.

According to the updated guidelines, women ages 21 through 29 should be screened with a Pap smear every 3 years. Women ages 30 through 65 can then be screened every 5 years with Pap smear and HPV cotesting or every 3 years with a Pap smear alone.

Cytologic diagnoses of the $\leq 20$-year age group were compared to the two older subsets. Diagnoses of high-grade squamous intraepithelial lesions (HSIL) were stratified by age group, and comparisons were made using the Pearson chisquare test. Statistical significance was declared at $p<0.05$. After a cytologic diagnosis of HSIL was made, patients who presented for follow-up underwent colposcopic directed biopsy with a cervical conization required in certain cases.

\section{Results}

A total of 19,481 Pap smear diagnoses were identified and reviewed: 2,229 diagnoses in the $\leq 20$-year age group, 5,294 diagnoses in the 21-30-year age group, and 11,958 diagnoses in the $>30$-year age group (Fig. 1). All HSIL cases from the $\leq 20$ year-age groups represented individual patients.

High-grade squamous intraepithelial lesions (HSIL) were diagnosed in thirty-six $(1.62 \%)$ patients $\leq 20$ years of age. The prevalence of HSIL diagnoses in the $\leq 20$-year age group was significantly ( $p=0.0478$ ) higher than the prevalence of diagnoses in the $>30$-year age group. While the prevalence of HSIL diagnoses in the $\leq 20$-year age group was higher than that of the 21-30-year age group (73 [1.38\%] patients), this increase was not statistically significant $(p=0.458)$. When compared to the national percentile reporting prevalence as per the College of American Pathologists in 2010, the prevalence of HSIL among the adolescent population in this study exceeds the 95th percentile of the national average of $1.5 \%$ [5]. Diagnoses of ASCUS, LSIL, and HSIL were highest in 
Fig. 1 Pap smear diagnosis review stratified by age group. The total numbers of Pap smear diagnoses that were reviewed for the study are presented by age category

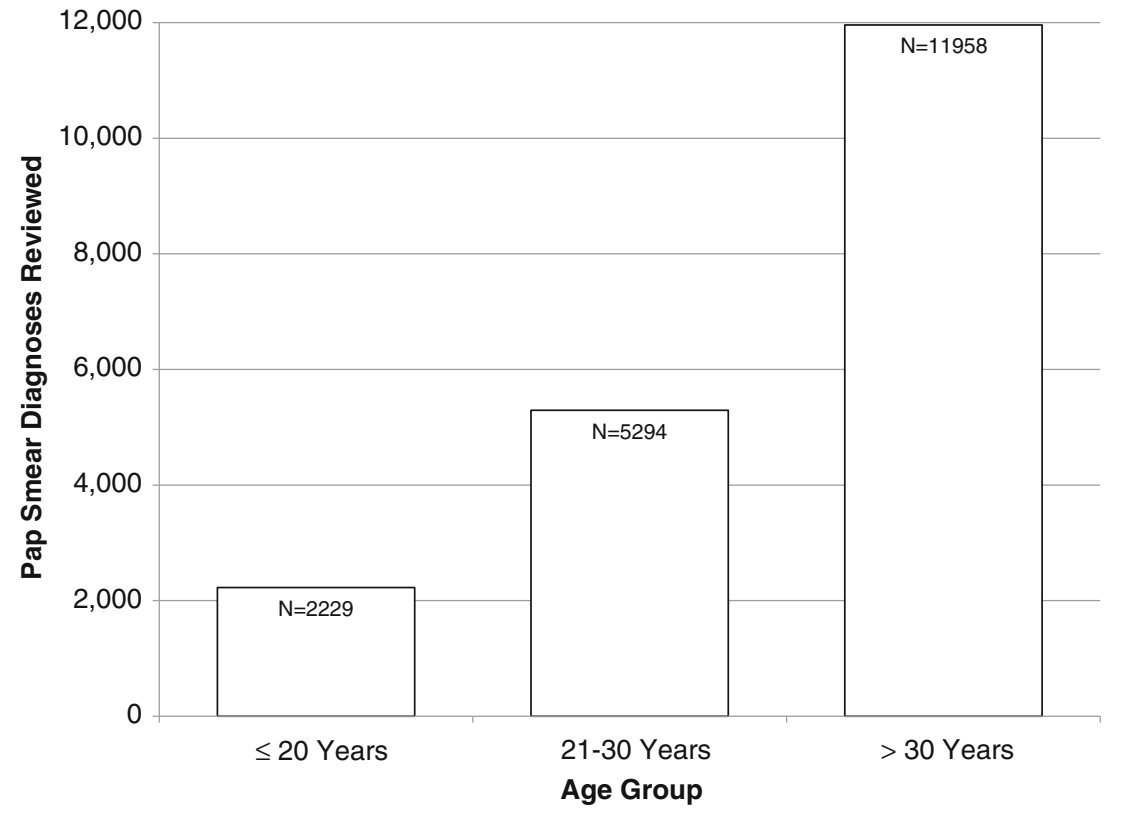

the $\geq 20$ age group compared to diagnoses among the 21-30year age group and $>30$-year age group (Table 1 ).

Of the thirty-six HSIL diagnoses in the $\leq 20$-year age group, $19(52.8 \%)$ patients were lost to follow-up. Of the seventeen $(47.2 \%)$ patients who had follow-up, fourteen underwent a colposcopic guided biopsy and three had cervical conization procedures. Follow-up testing indicated that no patients had any evidence of malignancy. Eleven $(64.7 \%)$ patients had a diagnosis of HSIL, four $(23.5 \%)$ patients had a diagnosis of LSIL, and two (11.8\%) patients had normal results on subsequent colposcopy or conization (Fig. 2). To determine which patients had follow-up or not, we reviewed pathology reports through the PowerPath system which shows all pathological diagnosis results since 1997. All colposcopic and cone biopsy slides post HSIL were pulled, reviewed, and evaluated by a board-certified pathologist with expertise in OB-GYN Pathology. Patients who were lost to follow-up or failed to show for colposcopic exam or cone biopsy were contacted. The duration of follow-up was based upon the date of primary diagnosis. For example, if the first pap diagnosis of HSIL was in 2000, records were examined from 2000 to 2012.

\section{Discussion}

This was a retrospective review of Pap smear diagnoses made at the Howard University Hospital between January 1997 and August 2010. This study makes an important contribution to the knowledge of cervical dysplasia in the African-American cohort, a population generally underrepresented in research, noncompliant, and at high risk for disease in the literature. When looking at the $\leq 20$-year age range with HSIL diagnosis, all patients except for one were African-American. The one patient that was not African-American was Hispanic. The age range of these patients were between 15 and 20 years of age. Research presented at the American Association for Cancer Research's 103rd Annual Meeting suggested a biological basis for the increased risk for cervical cancer seen in Black women, although the mechanism is unknown.

The Carolina Women's Care Study in 2004 evaluated the extent of HPV infection and persistence among college-aged women attending in the University of South Carolina.

In this longitudinal study, 467 study participants (70\% White, $24 \%$ Black) received biannual pelvic exams while
Table 1 Cervical cytology findings by age group

*Unsatisfactory for evaluation represent Pap smears with insufficient cellularity, poor cellular preservation or obscuring inflammation that preclude proper evaluation

\begin{tabular}{llll}
\hline Diagnosis & $\begin{array}{l}\leq 20 \text {-year age group } \\
(N=2,229)\end{array}$ & $\begin{array}{l}21-30 \text {-year age group } \\
(N=5,294)\end{array}$ & $\begin{array}{l}>30 \text {-year age group } \\
(N=11,958)\end{array}$ \\
\hline HSIL (\%) & $1.62 \%$ & $1.38 \%$ & $1.10 \%$ \\
LSIL (\%) & $7.00 \%$ & $3.27 \%$ & $1.40 \%$ \\
Atypical squamous cell (\%) & $8.17 \%$ & $6.83 \%$ & $3.73 \%$ \\
$\begin{array}{l}\text { Negative: intraepithelial lesion } \\
\quad \text { or malignancy (\%) }\end{array}$ & $82.23 \%$ & $87.60 \%$ & $92.75 \%$ \\
$\begin{array}{l}\text { Unsatisfactory for Evaluation* } \\
\text { n }\end{array}$ & $0.98 \%$ & $0.92 \%$ & $1.02 \%$ \\
\hline
\end{tabular}


Fig. 2 Follow-up pathology results of patients in the $\leq 20$-year age group. Where available, pathology results were tracked for patients in the $\leq 20$-year age group with diagnosis of HGSIL

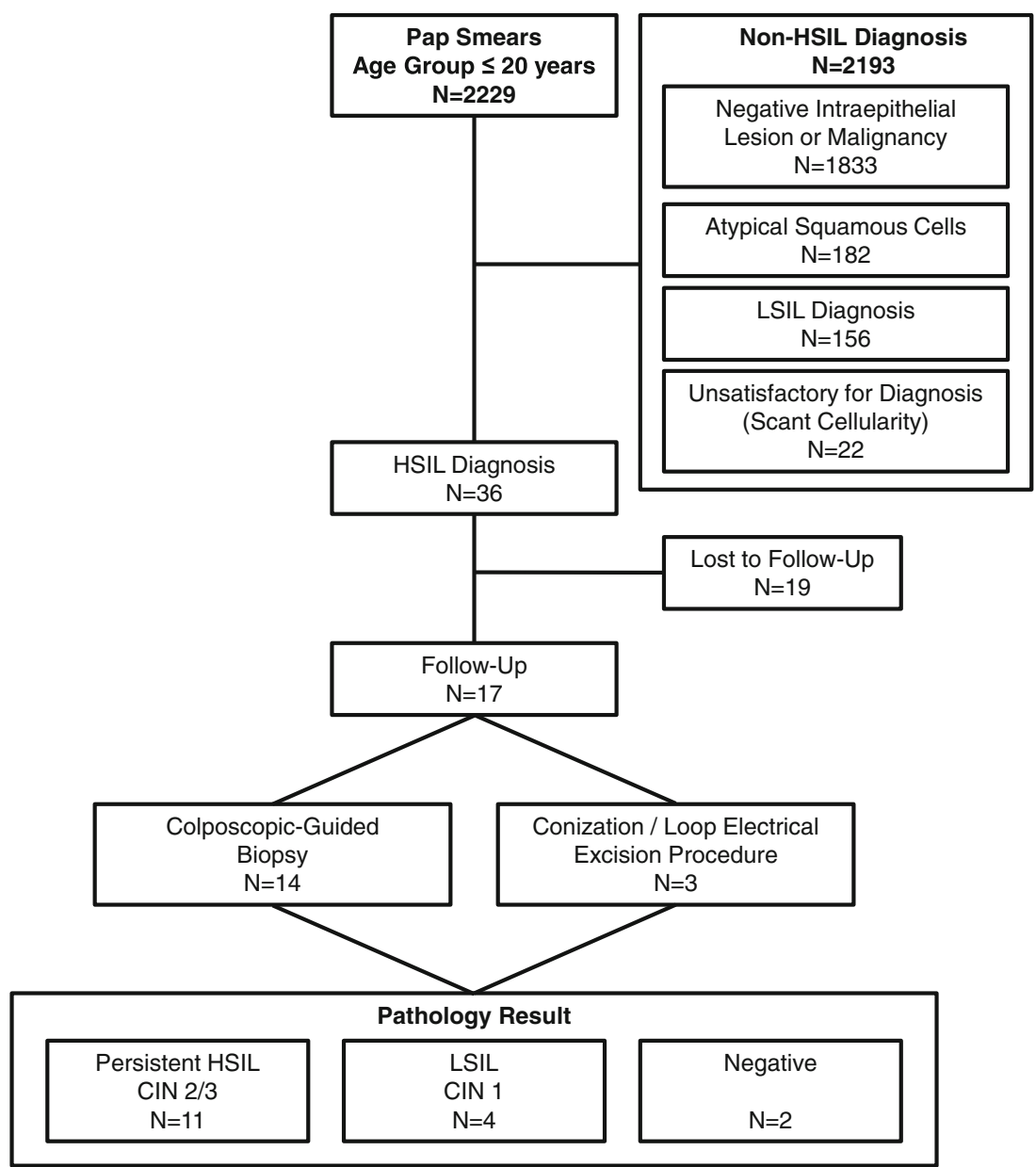

they attended in the university [6]. The researchers found that the study participants had similar demographic characteristics and similar behaviors associated with increased risk for cervical lesions, such as age at sexual debut and average number of sexual partners. Despite these similarities, however, results showed that Black women cleared the virus much slower than White women. The median duration of high-risk HPV infection was about 12 months in White women and approximately 18 months in Black women. At 24 months after infection with high-risk HPV, $56 \%$ of Black women remain infected, compared with $24 \%$ of White women [6]. In this study, the researchers also compared Pap test results. Black women were 1.7 times more likely to have an abnormal Pap test than White women (10.0 vs $6.2 \%)$. These results included the combined incidence of low-grade and high-grade squamous intraepithelial lesions [6].

Across specialties and disciplines, minority groups tend to have a higher rate of noncompliance. When adjusted for age and sex, there was a higher frequency of noncompliance among the Black and Hispanic subjects who were on lipid-lowering medications [7]. Nonadherence to anti- retroviral medications was significantly $(p<0.05)$ associated with the Black race and Hispanic ethnicity [8]. New recommendations for screening make effective strategies for increasing compliance imperative.

In 2009, ACOG, ACS, and the USPSTF revised their guidelines for cervical cancer screening by recommending initiating screening at 21 years of age [9]. Previous guidelines commenced cervical cancer screening at 18 years of age or at 3 years after onset of sexual intercourse (whichever was first). This change was prompted by data suggesting a low incidence of cancer in younger women. Daring to challenge this notion, Wright et al. reported high-grade abnormalities among $18 \%$ of 646 adolescent women $\leq 18$ years of age with cytologic abnormalities in 2003 . After 36 months, $50.9 \%$ of the HSIL cohort was found to have a high-grade abnormality. At 36 months, $31 \%$ of HSIL subjects had progressed to CIN 3. From this study, it was concluded that adolescents with LSIL and HSIL cytology are at significant risk for progression to high-grade cervical abnormalities. The study further revealed that the prevalence of development of high-grade cervical abnormalities in adolescents mimics that reported for adults 
[10]. As a result, adolescents with cytologic abnormalities mandate close follow-up [10]. Of 510 adolescent patients identified by Moore, et al. in 2007, $53 \%$ presented with findings of HSIL on cytology. $32 \%$ of those patients were found to have CIN 2 at the time of directed biopsy. $18 \%$ of patients were found to have CIN 3 at the time of directed biopsy. Interestingly, among adolescents referred for colposcopy with either atypical or low grade cytologic findings, $30 \%$ were found to have $\mathrm{CIN} \geq 2$ [10]. These findings supported continued vigilance in the evaluation of adolescents with cervical dysplasia.

Our study was limited by its retrospective nature and the fact that data extraction was exclusively from the university pathology reports. Therefore, several factors that could have contributed to the increased prevalence of HSIL in the study population, including exposure to HPV, history of past or current smoking, history of sexually transmitted infections such as human immunodeficiency virus (HIV), increased number of sexual partners, and history of contraceptive use, were precluded from investigation. Loss to follow up is a significant concern in this patient population which is reflected in our data. Though an assumption can be made that a percentage of our study patients may have followed up with primary care physicians or health departments outside of our institution, many of the patients in this age group are noncompliant and simply may not return for appropriate follow-up management.

\section{Conclusion}

Data from this study supported previously published reports of a high prevalence of HSIL in the adolescent population [10-12]. This projected highlighted the African-American population, a cohort that is underestimated in research, and, for which, additional studies are needed. Furthermore, the work showed that, although the prevalence of cervical dysplasia may be high, malignant transformation in the adolescent population is relatively low. As such, we agree with the current guidelines enforced by ACOG, ACS, and USPSTF.

\section{Compliance with Ethics Guidelines}

Conflict of Interest Authors Kevin S. Smith, Vanessa J. McDonald, and Babak Shokrani declare that they have no conflict of interest.
Human and Animal Rights and Informed Consent All procedures followed were in accordance with the ethical standards of the responsible committee on human experimentation (institutional and national) and with the Helsinki Declaration of 1975, as revised in 2000 (5). Informed consent was obtained from all patients for being included in the study.

\section{References}

1. Wright Jr TC, Cox JT, Massad LS, et al. for the 2001 ASCCP- sponsored Consensus Workshop 2001 consensus guidelines for the management of women with cervical intraepithelial neoplasia. Am J Obstet Gynecol. 2003;189: 295-304.

2. Howlader N, Noone AM, Krapcho M, Neyman N, Aminou R, Altekruse SF, et al., eds. SEER cancer statistics review, 1975-2009 (vintage 2009 populations). Bethesda (MD): National Cancer Institute; 2012. Available at: http://seer.cancer.gov/csr/1975_2009_ pops09. Retrieved November 29, 2013.

3. Watson M, Saraiya M, Benard V, Coughlin SS, Flowers L, Cokkinides V, et al. Burden of cervical cancer in the United States, 1998-2003. Cancer. 2008;113(suppl):2855-64.

4. Wright JD, Davila RM, Pinto KR, Merritt DF, Gibb RK, Rader JS, et al. Cervical dysplasia in adolescents. Obstet Gynecol. 2005;106: $115-20$.

5. College of American Pathologists (CAP) Cytopathology Checklist. http://www.cap.org. Accessioned 01/21/2012.

6. Banister CE, Messersmith AR, Chakraborty H, Wang Y, Spiryda LB, Glover SH, et al. HPV prevalence at enrollment and baseline results from the Carolina Women's Care Study: a longitudinal study of HPV persistence in women of college age. Int J Womens Health. 2013;5:379-88.

7. Kaplan RC, Bhalodkar NC, Brown EJ, White J, Brown DL. Race, ethnicity, and sociocultural characteristics predict noncompliance with lipid-lowering medications. Prev Med. 2004;39(6):1249-55.

8. Sullivan PS, Campsmith ML, Nakamura GV, Begley EB, Schulden J, Nakashima AK. Patient and regimen characteristics associated with self-reported nonadherence to antiretroviral therapy. PLoS One. 2007;2(6):e552.

9. Cervical Cytology Screening. ACOG Practice Bulletin No. 109. American College of Obstetricians and Gynecologists. Obstet Gynecol 2009.

10. Moore K, Cofer A, Elliot L, Lanneau G, Walker J, Gold MA. Adolescent cervical dysplasia: histologic evaluation, treatment, and outcomes. Am J Obstet Gynecol 2007;197: 141.e1-141.e6.

11. Moscicki AB, Shiboski S, Hills NK, Powell KJ, Jay N, Hanson EN, et al. Regression of low-grade squamous intra-epithelial lesions in young women. Lancet 2004;364:1678-83.

12. Fuchs K, Weitzen S, Wu L, Phipps MG, Boardman LA. Management of cervical intraepithelial neoplasia 2 in adolescent and young women. J Pediatr Adolesc Gynecol 2007;20:269-74. 